\title{
Der peinliche Erdenrest
}

\author{
Hermeneutische Dimensionen einer unbeschreiblichen \\ Erlösung des Geistes
}

Conrad Mattli

»Uns bleibt ein Erdenrest
Zu tragen peinlich,
Und wär er von Asbest
Er ist nicht reinlich. «

Mitgeteilte Peinlichkeiten sagen gemeinhin nicht nur darüber etwas aus, was dem jeweiligen Sprecher gerade peinlich ist, über den Gegenstand der Peinlichkeit, sondern auch über die subjektiven Bedingungen, die gegeben sein müssen, damit genau dieser Gegenstand als peinlich wahrgenommen werden kann. Der jeweilige Gegenstand der Peinlichkeit verrät so mitunter die Verfasstheit des Subjekts, welches die Peinlichkeit verspürt. Im Falle der Erlösungsszene in Goethes Faust II, wo die vollendeteren Engel den eingangs zitierten Vers sprechen, müssen wir auf eine Sphäre aufmerksam werden, in der der menschliche Erdenrest Fausts zum Gegenstand der Peinlichkeit wird. Wie können wir, die wir für gewöhnlich nicht dem geistigen Stande Goethes und Fausts angehören, verstehen, welches Kräftespiel von Hybris und Demut nötig ist, um mit der Faustdichtung auf Augenhöhe zu treten ? $^{2}$ Indem wir sie hermeneutisch reflektieren, ist die Antwort dieses Essays. Denn die Schlussszene des Faust II kann als Allegorie des Schicksals aller hermeneutischen Reflexion gelesen werden. Hermeneutisch denken heisst im gegebenen Kon-

\footnotetext{
1 So reden die vollendeteren Engel im Faust II, 5. Akt, "Faustens Unsterbliches tragend« (in: Johann Wolfgang Goethe, Faust. Der Tragödie Zweiter Teil, Stuttgart 2001, 215). In einem Paralipomena zur Szene aus dem Urfaust spricht Goethe statt vom "Unsterblichen" von der "Entelechie» Fausts. (Vgl. Johann Wolfgang Goethe, Faust. Der Tragödie erster und zweiter Teil. Urfaust, hg. v. Erich Trunz, München 1999, 730.) Der Begriff meint in Anlehnung an die aristotelisch-naturphilosophische Prägung hier die Vollendung eines inhärierenden Wesenszuges im Ganzen von Fausts Person, seine Entmaterialisierung im Zuge der Selbststeigerung zur reinen Tat, die als selbstbezügliche Form, als reiner Geist auf Welt und deren Stoff zu verzichten gelernt haben will.

2 Die exegetischen Stellen sind im Folgenden von den Betrachtungen Max Kommerells inspiriert, vor allem vom Aufsatz Faust II: Letzte Szene betreffend.Vgl. MaxKommerell, Geist und Buchstabe der Dichtung, Frankfurt a.M. 1956, 112-131.
} 
text, mit jener Peinlichkeit der Materialität auf vertrautem Fusse zu stehen. Denn solches Denken findet sich stets in einer Ruhelosigkeit zwischen den Reichen der Idealität geistigen Sinnes und der Materialität irdischen Wortes wieder - nicht unähnlich der Seele Fausts. Zugegeben, die Begrifflichkeit ist jetzt selbst schon schwer beladen. Aber es lässt sich beim Streifen der Geist-Körper-Thematik kaum auf beladene Begriffe verzichten. So viel sei mahnend vorweggenommen: Bei der Übertragung schwer beladener Begriffe in die ideelle Sphäre der hermeneutischen Reflexion sollen diese auch hinreichend >übertragen verstanden werden. Nur so eröffnet sich uns die hermeneutische Dimension der Peinlichkeit der Materialität, die zu kennzeichnen ich mich im Folgenden anschicke.

\section{Der höchste Augenblick}

Die Bekundung der Peinlichkeit des Erdenrests geht auf der Erzählebene mit der himmlischen Adelung Fausts bei seinem Tode einher. Dieser Tod ist Allegorie und Ereignis der Synthese seiner zweigeteilten Seele ("Zwei Seelen wohnen, ach! in meiner Brust $\left.[\ldots] \ll^{3}\right)$ - vereinigt durch den reinen Selbstbezug menschlicher Idealität. Dieses Ereignis - wie auch die subjektive Verfasstheit des Subjekts der Peinlichkeit - wird durch den Text nicht explizit benannt, sondern über den indirekten Weg der Erzählung der Elevation von Fausts Seele als Vollzug der göttlichen Gnade vermittelt. Das noch in der Selbstfixiertheit faustischer Hybris zentrierende erste Stück der Tragödie wird hierbei durch eine neu gewonnene Passivität kontrastiert, welche den lange vorweggenommenen Augenblick des Todes (»Und abgestreift den Erdensohn [...]«") kennzeichnet. Die Übernahme der faustischen Innerlichkeit durch ein Anderes findet dann Ausdruck in dem angesprochenen Peinlichkeitsverhältnis, das als solches nicht ohne den Einbruch des Fremden hergestellt würde. Man kann vielleicht sogar sagen, das Todesereignis werde bei aller Unbeschreiblichkeit durch die peinliche Fremdheit des leiblichen Erdenrests überhaupt erst hinreichend angezeigt. Selbst für den Fall nämlich, dass dieser Erdenrest unvergänglich ("von Asbest«) wäre,

\footnotetext{
3 Vgl. Johann Wolfgang Goethe, Faust. Der Tragödie Erster Teil, Stuttgart 2000, 33 : "Zwei Seelen wohnen, / ach! in meiner Brust, / Die eine will sich von der andern trennen; / Die eine hält, in derber Liebeslust, / Sich an die Welt mit klammernden Organen; / Die andre hebt gewaltsam sich vom Dust / Zu den Gefilden hoher Ahnen."

4 Goethe, Faust. Der Tragödie Erster Teil, 20.
} 
würde er aus der Sphäre vollkommener Vergeistigung aufgrund seiner Zugehörigkeit zur sanderen Seinssphäre der Materialität als "nicht reinlich" herausfallen. Damit ist der Sterbeakt als Übertritt in ein anderes $>$ Reich $\measuredangle$ zu verstehen, von wo aus der Erdenrest erst als peinlich gelten kann und darf. Die Peinlichkeitsbeziehung zeitigt hierbei eine unerwartete Hierarchie geistiger Vervollkommnungsebenen, ähnlich wie dies später bei Nietzsches Stufenleiter vom Affen zum Menschen und zum Übermenschen geschieht, wo das "Gelächter" und die "schmerzliche Scham" den jeweiligen Blick von oben herab auszeichnen. ${ }^{5}$ Fausts Tod wird so als die eigentlich unmögliche (»unzulängliche») Seinsmöglichkeit eines Menschen eröffnet, der auf seinen Pfaden, vom "Ewig-Weiblichen « angezogen, mit der Kraft eines unbedingten Strebens in ebendiese Sphäre emporsteigt und am Ende durch die Gnade svon oben emporgehoben wird. ${ }^{6}$ Nur dort oben, wo der Sterbende bereits ganz Geist geworden ist, erlangt die Peinlichkeitsbekundung hinsichtlich des Erdenrests ihren vollen Sinn. Die letale Entmaterialisierung Fausts setzt schon bei seiner Erblindung ein, und der Zeitpunkt des Todes erweist sich dann als der 'höchste Augenblick ${ }^{7}$ (durch die körperliche Blindheit den ohnehin (temporal-)übertragenen Sinn des Wortes `Augenblick und mithin den des Todes noch steigernd) in einer selbstgeschaffenen, aber objektiv bedingten Illusion der freien Gesellschaft. Diese Vision ist Illusion, weil ihr jede Gegenwärtigkeit fehlt: Das Klingen der Spaten, welche in Fausts Wahrnehmung den "freien Grund « seines "paradiesisch Land « freilegen, ist in Wahrheit auf die Lemuren zurückzuführen, welche Fausts Grab graben. ${ }^{8}$ Der präzedenzlose Grad selbstbezüglicher Vergeistigung in solcher Illusion zeigt also den höchsten Augenblick Fausts als dessen Tod an. Die reine Tat seines Geistes ist in ihrer selbstbezüglichen Unbeschreiblichkeit primärer als das Wort des Dichters (- "Alles Vergängliche / Ist nur ein Gleichnis «"). Dies sind die Konsequenzen einer Auslegung des Faust, die dem hermeneutischen Wink folgt, demgemäss der Fokus auf dem allegorischen Charakter der manifesten Erzählung liegen

\footnotetext{
5 Vgl. Friedrich Nietzsche, Also sprach Zarathustra. Ein Buch für Alle und Keinen, in: Ders., Kritische Studienausgabe (Bd. 4), hg. v. Giorgio Colli und Mazzino Montinari, Berlin/New York ${ }^{2}$ 1999, 14: „Was ist der Affe für den Menschen? Ein Gelächter oder eine schmerzliche Scham. Und ebendas soll der Mensch für den Übermenschen sein: ein Gelächter oder eine schmerzliche Scham."

6 Vgl. Goethe, Faust. Der Tragödie Zweiter Teil, 219.

7 Ebd., 204.

8 Vgl. ebd., 203.

9 Ebd., 219.
} 
solle ("Denn wir sind Allegorien / Und so solltest du uns kennen $\left.{ }^{10}\right)$. Folglich will das Kommende in den Skopus der Wendung smit anderen Worten gesetzt sein. `Mit anderen Worten « wird allerdings zu einer höchst fragwürdigen Wendung, sobald wir es, wie hier, mit eigentlich Unbeschreiblichem zu tun haben. Allerdings wird die Not der Fragwürdigkeit des Anderssagens des Unsagbaren ausgerechnet zur Tugend der Allegorie. Denn unser Gegenstand, die faustische Erlösung am Ende des zweiten Teils der Tragödie, wird im letzten Vers als derVollzug des Unbeschreiblichen benannt. (»Alles Vergängliche / Ist nur ein Gleichnis; Das Unzulängliche / Hier wird's Ereignis; / Das Unbeschreibliche, hier ist es getan; / Das Ewig-Weibliche / Zieht uns hinan. $\left.{ }^{11}\right)$ Jenes Unzulängliche, welches in Fausts Erlösung Ereignis wird, ist als dieVollendung einer Bewegung anzusehen, welche das gesamte Opus im Zeichen des sfaustischen Strebens; überwölbt: Fausts allseitiges Verlangen nach einer Anverwandlung von Welt und ihres Stoffes in die idealen Formen von Wissenschaft und Kunst - das unbedingte Vordringenwollen zum Wesenskern der Welt. Die Mitte, welche die Welt im Innersten zusammenhält, wird durch den Brennpunkt einer Synthese markiert, welche die Idealität eines zur Hybris der unbedingten Selbststeigerung drängenden Geistes mit der Materialität eines Körpers, der als solcher einer diesem Geist rätselhaften Welt angehört, vereinigt. Diese Mitte ist für sich nur indirekt ১beschreibbar`, da sie, würde sie denn hinlänglich beschrieben`, zeigen würde, was die Welt tatsächlich im Innersten zusammenhält und so die Grenzen der im Gleichnis verdichteten Welt überschritte. Das unbedingte Streben des faustischen Geistes ist am Ende paradoxerweise trotz des lange antizipierten Scheiterns an der unmittelbaren Wesenserkenntnis der Eintrittsgarant ins Himmelsreich und die Loslösung vom blutunterzeichneten Vertrag mit Mephistopheles. Fausts Vergeistigung ist damit ein Gleichnis für die Gleichniswerdung des Textes selbst. Mit anderen Worten: Der faustische Tod ist die mimetische Ereigniswerdung der Allegorese, die jederzeit ihren Erdenrest transzendiert, indem sie an ihm emporsteigt.

\footnotetext{
${ }^{10}$ Dies verkündet einer der Knaben im Faust II (Goethe, Faust. Der Tragödie Zweiter Teil, 28) und nimmt damit die Rolle der seligen Knaben im Erlösungsschluss vorweg, die als Boten himmlischer Gnade fungieren, weil sie aufgrund ihres frühen Todes ihres Erdenrests ohnehin schon ledig sind. Die reine Allegorizität findet dort ihren allegorischen Niederschlag in Gestalt der Knaben, die das reinliche Komplement zur irdisch erfahrenen Seele Fausts darstellen.

${ }^{11}$ Goethe, Faust. Der Tragödie Zweiter Teil, 219.
} 


\section{II. »Dass ich erkenne was die Welt / Im Innersten zusammenhält $[\ldots]$ « $^{12}$}

Kommen wir auf die bereits angedeutete Frage zurück, von welcher Verfasstheit ein Geist (die Idealität) überhaupt sein muss, um seinen Erdenrest (die Materialität) als "nicht reinlich und folglich "peinlich« zu begreifen. Hierbei verweise ich nun auf das Reich der Hermeneutik. Mich interessiert die hermeneutische Dimension des Problems von Idealität und Materialität, so wie es sich zum Beispiel in der Buchstabe-Geist-Problematik oder, allgemeiner und moderner, in der Hermeneutik des Verdachts ${ }^{13}$ niedergeschlagen hat. Denn das - wenn man so will - >klassische hermeneutische Problem von Buchstabe und Geist steht emblematisch für letzteres Problem als ein noch allgemeineres Problem des Denkens. Ich spreche vom Verdacht als Denkbewegung, die ihre Dynamik jeweils aus dem Hin und Her zwischen oberflächlicher Phänomenalität und untergründiger Materialität schöpft. Bezüglich der Geist-Gehirn-Thematik etwa besteht nach wie vor das Problem, dass das Denken in einem meist dualistisch ausgetragenen Streit um das Vorrecht des Realen Stellung beziehen muss - auch und gerade in Zeiten des Siegeszugs eliminativer Reduktionismen des Geistes auf seine materiellen Grundlagen. Die Auslotung des Menschseins zwischen einem Dasein in den himmlischen Sphären göttlicher Idealität und einem Dasein in einer Ansammlung von Staub fällt heute schnell in die Niederungen einer Körperlichkeit, die sich des Vorurteils des Mechanismus noch nicht entledigen konnte. Es ist weit eher zur Selbstverständlichkeit geworden, dass man sich des Geistes schämt, wenn der Wettlauf um die irdischste Erklärung der machina corporea erst einmal eröffnet ist. Die landläufige, die selbstverständliche Meinung spricht sich also gerade nicht im faustischen Gleichnis des peinlichen Erdenrests aus, sondern richtet sich viel lieber gegen den peinlichen Himmelsrest, den sie so nicht anders denn als Nachwirken eines toten Gottes verstehen kann. Was diese Selbstverständlichkeit allerdings aus dem Selbstverständnis ihrer Verfechter wegzensiert, ist die jederzeitige Möglichkeit des Heraustretens aus der Dialektik von profanem Maschinismus und sakraler Geistesbeschwörung im hermeneutischen Denken, genauer eben in der hermeneutischen Bewegung des Ver-

\footnotetext{
${ }^{12}$ Goethe, Faust. Der Tragödie Erster Teil, 13.

${ }_{13}$ Der Ausdruck entstammt bekanntlich dem Cuvre des französischen Philosophen Paul Ricœur. Er charakterisiert die Interpretationspraxis Freuds neben derjenigen von Marx und Nietzsche als "Schulen des Zweifels«. Siehe: Paul Ricœur, Die Interpretation. Ein Versuch über Freud, Frankfurt a.M. 1969, 41-49.
} 
dachts. DerVerdacht ist das Nicht-Verharren bei der Oberfläche und die Rückführung der Oberflächenphänomene auf die sie bedingende Ökonomie darunter - meist deren Materialität, wobei jener Verdacht, ist er erst einmal am Grund angelangt, nicht einfach wieder in Vertrauen umschlägt. Dass der Verdacht nicht das letzte Wort behält, wenn dadurch nur ein neues Positives an die Oberfläche träte, beweist etwa das Scheitern Freuds in einer letzthinnigen positiven Bestimmung der untergründigen Triebökonomie, ebenso wie die Verkomplizierung der Verdachtshermeneutik bei deren Urhebern selbst: bei Nietzsche durch die Konzeption einer Oberflächlichkeit aus Tiefe, bei Marx durch den möglichen, revolutionären Übergriff auf die ökonomische Basis und weiter bei Darwin, wo die evolutionäre Historizität aller phänomenalen Geistigkeit im Sinne einer kulturellen Einwirkung auf die evolutionäre Genese des Menschen auf die biologischen Grundlagen rückwirkt. Das Untergründige ist als postulierte Tatsache nie der hermeneutischen Weisheit letzter Schluss. DerVerdacht muss sich also ebenso wie als ein unbedingtes Streben als unaufhörliche Bewegung verstehen, im Sinne der hermeneutischen Dynamik der unabschliessbaren Interpretation. Damit entspricht derVerdacht aber gerade jenem Zug der faustischen Seele, welcher deren unaufhörlich strebendes Bemühen bezeichnet. Denn auch dieses Streben gelangt erst zu sich selbst, wo es sich als solches bejaht und folglich begriffen hat, dass es kein absolutes Positives zeitigen kann. Der Verdachtshermeneutiker ist stets nur so klug als wie zuvor, begreift er nicht, dass die Schule des Verdachts keine jenseitige oder verborgene Welt eröffnet, sondern lediglich einen neuen $\mathrm{Zu}$ gang zur sgleichen< Welt. Er betrachtet die Welt lediglich in neuem Abglanz. Das Oszillieren zwischen Geist und Material wird im Falle des Verdachts zwar meist in einer absteigenden, weil verneinenden Bewegung vollzogen. Allerdings soll damit nicht gesagt sein, dass die verdächtigende Reduktion der Idealität auf die Materialität einer blossen Verneinung und Abtragung der geistigen Phänomenalität gleichkommen muss. Als Denkbewegung bleibt die Bewegung der Verneinung einer ideellen Dynamik unterworfen und bezeugt die ubiquitäre Anwesenheit des Geistes - selbst in seiner äussersten Verneinung durch die Reduktion auf das Körperliche. Zu erkennen, was die Welt im Innersten zusammenhält, führt das faustische Denken also nicht an sich selbst vorbei. Erst wenn sich das Denken vor dem Spiegel hermeneutisch läutern lässt, das heisst, wenn es die Dynamik der Gegensatzkonstitution aus sich selbst heraus begreift, verlässt es die Dialektik von oben und unten, Himmel und Hölle. Nur so kann ja Faust dem Teufelspakt entkommen; nämlich dadurch, 
dass er nicht zum Antipoden, zu Gott, rennt, sondern in sich selbst den reinen Selbstbezug der Tat vollzieht. Der sterbende Faust ist das sich selbst einholende und allezeit selbst destabilisierende (weil entmaterialisierende) hermeneutische Subjekt. Natürlich bleibt das höchst private Erlebnis dieses Subjektes im Letzten >unbeschreiblich und kann durch die Erzählung, wie gesagt, nur indirekt vermittelt werden. Die Figuren der Büsserinnen, der Chorus Mysticus oder die drei Patres entspringen freilich, ebenso wie die Engel, einer Topographie des faustischen Denkraumes, den $>$ Bergschluchten seines Geistes, die er sich am Kern seiner Welt erschlossen hat. Weil sich Faust in seiner Selbstbeteiligung am Sein des Erzählten begriffen hat, muss die höchste Offenbarung ein Schauspiel bleiben. "Welch Schauspiel! aber ach! ein Schauspiel nur! ${ }^{14}$, hiess es noch im ersten Teil der Tragödie. Dem hermeneutisch sich läuternden Faust des zweiten Teils ist aber die bescheidende Erkenntnis zuteilgeworden, dass gerade der farbige Abglanz der Dinge das Leben verbürgt. ${ }^{15}$ Der schauspielhafte Schein des Seins ist so als dem (neu einem hermeneutischen Perspektivismus unterliegenden) Sein zugehörig erkannt worden. Denn es scheint zur nie enden wollenden Herausforderung für das Denken geworden zu sein, dass seine architektonisch bedingte, götterschaffende Neigung zum Ganzen hin mit der Einsicht in den Schein als die eigene Begrenztheit einhergehen muss. Das Denken streift das Ganze somit nur noch dann, wenn es seine Grenzen anzeigen kann, und es vermag erst dann das an Faust gerichtete Wort des Erdgeistes zu vernehmen, welches besagt: "Du gleichst dem Geist den du begreifst, / Nicht mir! " $^{16}$ So ist also die Perspektivität vom notwendigen Übel zur Bedingung der Möglichkeit innerster Welterkenntnis geworden.

\section{Schluss: Kennzeichnung der hermeneutischen Dimension}

Die Faust-Tragödie legt, wie mittlerweile evident geworden sein sollte, mit ihrer Allegorizität ein ungemein grosses hermeneutisches Potenzial an den Tag. Dieses Potenzial gilt es zum Schluss noch einmal zu entbergen, indem der faustische Tod als die Geburt des Sinnes aus dem Wort verstanden wird. Die Hermeneutik kann als der Vollzug eines in die Idealität des Sinnes strebenden Denkens

\footnotetext{
${ }^{14}$ Goethe, Faust. Der Tragödie Erster Teil, 15.

${ }^{15}$ Goethe, Faust. Der Tragödie Zweiter Teil, 6.

${ }^{16}$ Goethe, Faust. Der Tragödie Erster Teil, 17.
} 
verstanden werden, das sich seiner Gebundenheit an die Materialität des Wortes jederzeit bewusst sein muss. Der Sprung von der Hermeneutik ins Philosophische führt hier - in dezidiert unzeitgemässer Manier - den Glauben an die Ubiquität hermeneutischen Denkens in philosophischen Belangen als stille Prämisse mit sich. Dieser Sprung drängt sich allerdings geradezu auf, und wenn wir berücksichtigen, dass die Peinlichkeit des Erdenrests der Lebenswelt der Gefühle entnommen und ins abstrakte Reich der hermeneutischen Ausmessung des Verhältnisses von Idealität und Materialität übertragen wird, so springen wir aus der Idealität des hermeneutischen Reiches lediglich zurück in das Leben, wo die Notion der Peinlichkeit ihren eigentlichen Platz hat. Schauen wir aber, ob der Sprung überhaupt gelingt. Lässt sich von einer philosophischen Ubiquität des hermeneutischen Denkens sprechen, so offenbart sich gerade hier, im Widerstreit von Idealität und Materialität, deren volles Ausmass. In der Frage des ontologischen Vorrechts des Geistes oder des Körpers etwa ist es der hermeneutische Geist, der sich unmittelbarer als die eröffneten Gegensätze präsentiert, weil er das ontologische Medium der phänomenalen Welterschliessung bildet, in welchem die Gegensätze überhaupt zum Gegenstand werden können. Die Betonung der Identität des Geistigen mit den zugrundeliegenden körperlichen Prozessen indiziert meines Erachtens zuallererst die Distanz und Absurdität des Geistigen in Bezug auf jene Materialität des Körpers, die paradoxerweise für realer befunden wird. Das offenbare Ausbleiben des absoluten Evidenzerlebnisses im Falle Fausts ist sinnbildlich für jenes Schicksal der Absurdität, weil auch Letzterer sich, wie der mechanisierte Mensch, im unheimlichen Gewahrwerden der perspektivisch bedingten Distanz zu den festen Dingen heimisch fühlen lernen muss. Die hermeneutische Reflexion ist, wie das faustische Streben hin zum absoluten Wissen, durch ein ewiges Nicht-Ankommen in der Absolutheit des Sinnes gekennzeichnet. Das Scheitern an jener Unendlichkeit des absoluten Sinnes ist aber genau als ewiges Streben die Bedingung der faustischen Erlösung und gleichsam die Bedingung der Einlösung des hermeneutischen Telos. Dieses faustische Verstehen ist die Vollendung des Weges einer Entelechie der Selbstbesinnung. (»Wer immer strebend sich bemüht / Den können wir erlösen. ${ }^{17}$ ) Auf das Geschäft der Hermeneutik übertragen, legt die Allegorie des Todes und die Pein der Materialität also eine bejahende Rückberufung auf die Dynamik des hermeneutischen Denkens selbst nahe. Dieses Denken erlöst den Sinn der materialen Gegenständlichkeit in einem unaufhörlichen

17 Goethe, Faust. Der Tragödie Zweiter Teil, 214. 
Kreisen um sich selbst, weil es den peinlichen Erdenrest stets in die $\mathrm{zu}$ verstehende Welt hineinträgt und von dort wieder zu sich selbst zurückkehrt. Der Sinn bleibt als Geist nur dann lebendig, wenn er in seinem Emporstreben weg vom Erdenrest als dem sterbenden Buchstaben begriffen wird und nicht als absolut vom sterbenden Buchstaben. Der Geist bleibt lebendig, solange der Buchstabe nie aufhört zu sterben. Es liegt an uns, jenen Geist immer wieder von Neuem zu erlösen. Vielleicht wird das Denken dann auch einmal wieder in jene Höhen vordringen, von wo aus der Erdenrest in der ihm anhaftenden Peinlichkeit - ja, diese Peinlichkeit vielleicht sogar als Auszeichnung begriffen werden darf. Das Gefuihl der Peinlichkeit hinsichtlich des irdischen Restes des Buchstabens wird dabei das Gelingen der Erlösung des Sinnes bezeugen. Dies wird allerdings nicht geschehen, solange das immer schon auf die Erlösung des Sinnes aus dem Stofflichen abzweckende Denken nicht begonnen hat, sich nach dem Vorbild des goetheschen Faust in seiner hermeneutischen Schöpfungspotenz zu begreifen. Nur so können wir über die unvereinbaren Gegensätze, die unser ptolemäisches Gemüt immerfort durchdringen, vielleicht einmal verfügen lernen.

- Conrad Mattli ist Masterstudent der Philosophie an der Johann Wolfgang Goethe-Universität in Frankfurt am Main. Er hat seinen Bachelorabschluss an der Universität Zürich in Philosophie, Allgemeine Vergleichende Literaturwissenschaft und Hermeneutik erworben. 